\title{
Molecular-based classification algorithm for endometrial carcinoma categorizes ovarian endometrioid carcinoma into prognostically significant groups
}

Carlos Parra-Herran ${ }^{1,2}$, Jordan Lerner-Ellis ${ }^{2,3,4}$, Bin $\mathrm{Xu}^{1,2}$, Sam Khalouei $^{3}$, Dina Bassiouny ${ }^{1,5}$, Matthew Cesari ${ }^{1,2}$, Nadia Ismiil ${ }^{1,2}$ and Sharon Nofech-Mozes ${ }^{1,2}$

${ }^{1}$ Department of Anatomic Pathology, Sunnybrook Health Sciences Centre, Toronto, ON, Canada; ${ }^{2}$ Department of Laboratory Medicine and Pathobiology, University of Toronto, Toronto, ON, Canada; ${ }^{3}$ Department of Pathology and Laboratory Medicine, Lunenfeld-Tanenbaum Research Institute, Sinai Health System, Toronto, ON, Canada; ${ }^{4}$ Ontario Institute for Cancer Research, Toronto, ON, Canada and ${ }^{5}$ Department of Pathology, Mansoura University, Mansoura, Egypt

The Cancer Genome Atlas classification divides endometrial carcinoma in biologically distinct groups, and testing for p53, mismatch repair proteins (MMR), and polymerase $\varepsilon$ (POLE) exonuclease domain mutations has been shown to predict the molecular subgroup and clinical outcome. While abnormalities in these markers have been described in ovarian endometrioid carcinoma, their role in predicting its molecular profile and prognosis is still not fully explored. Patients with ovarian endometrioid carcinomas treated surgically in a 14-year period were selected. Only tumors with confirmation of endometrioid histology and negative WT1 and Napsin-A were included. POLE mutational analysis and immunohistochemistry for p53, MLH1, MSH2, MSH6, and PMS2 was performed in formalin-fixed, paraffin-embedded tissue. Following the molecular classifier proposed for endometrial carcinoma (Br J Cancer 2015;113:299-310), cases were classified as POLE mutated, MMR abnormal, p53 abnormal, and p53 wild type. Clinicopathologic information was recorded, including patient outcome. In all, 72 cases were included, distributed as follows: 7 (10\%) POLE mutated; 6 (8\%) MMR abnormal; 17 (24\%) p53 abnormal; and $42(58 \%)$ p53 wild type. The molecular classification correlated with disease-free survival in multivariate analysis $(P=0.003)$, independently of tumor grade and stage. Correlation with overall survival approached statistical significance $(P=0.051)$. POLE-mutated and MMR-abnormal tumors had excellent survival, whereas p53-abnormal tumors had significantly higher rates of recurrence and death. Ovarian endometroid carcinoma can be classified in clinically meaningful subgroups by testing for molecular surrogates, akin to endometrial cancer. MMR and POLE alterations seem to identify a subset of ovarian endometrioid carcinomas with excellent outcome; conversely, abnormal p53 carries a worse prognosis. In the era of personalized medicine, the use of these markers in the routine evaluation of ovarian endometrioid tumors should be considered.

Modern Pathology (2017) 30, 1748-1759; doi:10.1038/modpathol.2017.81; published online 4 August 2017

In 2013, The Cancer Genome Atlas (TCGA) identified four genomic groups of endometrial carcinoma that correlate with clinical outcome. ${ }^{1}$ The proposed classification has since then provided important insight into the biology of this prevalent gynecologic

Correspondence: Dr C Parra-Herran, MD, Department of Laboratory Medicine and Pathobiology, University of Toronto, and Department of Anatomic Pathology, Sunnybrook Health Sciences Centre, 2075 Bayview Avenue, Room E4-27a, Toronto, ON M4N 3M5, Canada.

E-mail: carlos.parraherran@utoronto.ca

Received 22 March 2017; revised 6 May 2017; accepted 7 May 2017; published online 4 August 2017 disease and tools to better assess the prognosis and therapeutic options for endometrial cancer patients. The classification describes a novel subset of tumors with mutations in the polymerase $\varepsilon(P O L E)$ exonuclease domain and a consequent 'ultramutated' phenotype, which carry a favorable prognosis regardless of the tumor grade or histology. In contrast, tumors classified as 'copy number high' are associated with poor prognosis. The 'microsatellite instability' and 'copy number low' groups have intermediate disease-free survival.

On the basis of the above associations, determining the molecular group in patients diagnosed with 
endometrial carcinoma is clinically relevant. To achieve this goal cost-effectively, ancillary testing using formalin-fixed, paraffin-embedded tumoral tissue can serve as a surrogate of the genomic profile, and thus the molecular group. ${ }^{2} \mathrm{~A}$ molecular-based classifier was recently proposed and validated by Talhouk et al, ${ }^{3,4}$ based on the TCGA classification using POLE mutational analysis as well as immunohistochemistry for p53 and mismatch repair proteins (MLH1, MSH2, MSH6, and PMS2). This algorithm not only correlated with clinical outcomes similarly to the TCGA classification in univariate and multivariate analysis but also improved patient prognostic stratification when combined with established clinicopathologic risk factors. The main advantage of using such a tool is its potential feasibility, as immunohistochemical testing for p53 and mismatch repair proteins is already widely available in clinical laboratories.

Ovarian endometrioid carcinoma is a frequent primary epithelial malignancy of the ovary, closely related to endometrioid endometrial carcinoma. The architectural and cytologic morphology and the range of variant differentiation (squamous, mucinous, and secretory) largely overlap in both tumors. In addition, endometriosis is frequently found in patients with ovarian endometrioid carcinoma and is regarded as a non-obligatory precursor lesion.

The morphologic and biologic similarities between ovarian and endometrial endometrioid carcinomas suggest similarities in the genomic landscape. While abnormalities in p53, MMR, and POLE have been described in ovarian endometrioid carcinoma, the role of these markers in predicting the molecular profile and thus the prognosis is still not fully explored. We hypothesize that ovarian endometrioid carcinomas can be classified in clinically meaningful subgroups by testing for these markers as molecular surrogates, akin to endometrial cancer.

\section{Materials and methods}

This study was approved by the Research Ethics Boards at Sunnybrook Health Sciences Centre and Mount Sinai Hospital in Toronto, ON, Canada.

\section{Case Selection and Review}

In-house ovarian cancer patients treated surgically at Sunnybrook Health Sciences Centre between 2000 and 2013 were retrieved from the Anatomic Pathology database. After exclusion of metastatic tumors, cases with controversial site of origin, and cases with minimal microscopic disease post neoadjuvant chemotherapy, 702 patients with primary carcinoma were available. Tumors diagnosed as endometrioid type per World Health Organization criteria were further selected; cases with synchronous myoinvasive endometrial carcinoma or endometrioid metastases to ovary from endometrium on report were excluded. Follow-up data were collected from the electronic patient record, including time to recurrence, last date of follow-up, and status at follow-up. Histologic diagnosis of endometrioid carcinoma was confirmed in all cases by independent review of all available hematoxylin-and-eosinstained slides by one fellow and at least two gynecologic pathologists; only cases with full consensus on histotype were included. In addition, presence of at least one confirmatory endometrioid feature was required for inclusion: background of endometriosis and/or endometrioid adenofibroma or metaplasia (squamous, mucinous, and/or secretory). Further confirmation of the diagnosis involved the use of immunohistochemistry (see section below). Histologic grade and pathologic staging were recorded according to the international Federation of Gynecology and Obstetrics (FIGO) grading and staging systems.

\section{Immunohistochemistry}

Two $1 \mathrm{~mm}$ cores representative of the tumor were extracted from paraffin blocks and arrayed using a tissue arrayer (Beecher Instruments, Silver Spring, MD, USA). Immunohistochemistry was performed on formalin-fixed, paraffin-embedded tissue microarray sections with monoclonal antibodies against p53 (DO7; Ventana), MLH1 (G168-15; Bio Care Medical), MSH2 FE11; Calbiochem), MSH6 (BC/44; Becton Dickinson), PMS2 (A16-4; Becton Dickinson), WT1 (6 F-H2; Ventana), Napsin-A (MRQ-60, Ventana), ARID1A (HPA005456; dilution 1:100; Sigma Aldrich), and PTEN (9559; dilution 1:25; Cell Signaling). A positive control was included in every run. All stains were reviewed independently by at least two observers. P53 was interpreted as abnormal (overexpressed with strong nuclear staining in more than $75 \%$ of tumor cells, or null with absent staining) or normal (nuclear staining with variable intensity and patchy distribution). When the two cores from any given tumor were discordant, a whole slide section was re-stained. Mismatch protein stains were scored as normal (retained nuclear staining) or abnormal (absent staining or very weak nuclear staining in $<5 \%$ of tumor cells). Cases without immunoreactive stromal cells in the microarray were further tested on whole tissue sections. WT1 and Napsin-A stains were interpreted as negative (absent staining) or positive (any intensity). ARID1A and PTEN stains were interpreted as retained (any intensity) or lost expression (absent staining).

\section{POLE Mutational Analysis}

DNA was isolated from microdissected tumor tissue (curls) obtained from block areas with high tumor cellularity ( $80 \%$ or more) marked by a pathologist. DNA extraction was carried out using the QIAsymphony DSP DNA Mini kit 192 Version-1 and 
included a ProK digest buffer ATL (Qiagen, Hilden, Germany). DNA was amplified by PCR for exons 9, 11, 13, and 14 of the POLE gene on chromosome 12 (hg19 refGene: NM_006231). DNA dideoxycapillary-based sequencing was performed on an ABI 3730 DNA Analyzer. Primer sequences are available on request. Analysis included the coding regions and splice sites. Data analysis was carried out using Mutation Surveyor software (Softgenetics, PA, USA). Variant classification was based on the 2015 American College of Medical Genetics and Genomics guidelines for interpreting sequence variants and followed assessment procedures described by the Canadian Open Genetics Repository. ${ }^{5,6}$

\section{DNA Molecular Classification Model}

A model for molecular classification of endometrial carcinoma was used as proposed by Talhouk et al based on survival analyses, $C$-index, anticipated clinical benefit of testing, and cost/accessibility of methods. ${ }^{3}$ Tumors were divided into four groups based on POLE, MMR, and p53 results as follows (Figure 1):

- POLE-mutated group: any case with a pathogenic or likely pathogenic POLE mutation.

- MMR-deficient group: any remaining case with abnormal expression of any MMR marker.

- P53 abnormal: any remaining case with an abnormal p53 expression.

- P53 wild type: all remaining cases with absence of POLE, MMR, or p53 abnormalities as described above.

\section{Statistical Analysis}

For analysis purposes, time of recurrence and time of death were used to estimate disease-free and overall survival, respectively. The time to recurrence was defined from the date of primary surgery to the date of first recorded recurrence by imaging or tissue diagnosis. Likewise, time of death was defined from the date of primary surgery. Prognostic value of the molecular classifier categories and other variables was determined using univariate log rank test against overall, disease-free and disease-specific survival. Multivariate Cox proportional hazard regression model analysis was subsequently conducted to weight the molecular classifier, and other clinical and pathologic variables that were significant on univariate analysis (ie, FIGO stage, tumor grade, lymph node status and adjuvant therapy). The correlation between molecular groups and various clinicopathologic characteristics was calculated using appropriate statistical tests, ie, $\chi^{2}$-test for categorical variables and one-way ANOVA for continuous variables. All statistical analyses were performed using the SPSS software 24.0 (IBM Corporation, New York, NY, USA). $P$-values $<0.05$ were considered statistically significant.

\section{Results}

A total of 97 cases with initial diagnosis of ovarian endometrioid carcinoma were identified. Of these, 16 were excluded after secondary review and immunohistochemistry: 15 tumors were re-classified as serous based on morphologic features and WT1 positivity, and 1 was re-classified as clear cell based on morphology and Napsin-A positivity. Of the remaining 81 cases, 9 were further excluded due to lack of clinical follow-up (6 patients) or failure to extract DNA for molecular testing (3 patients). The remaining 72 cases comprise the final cohort of the study. Median patient age was 53 years (mean 55, range $27-91)$. A total of 6 patients (8\%) underwent salpingooophorectomy only; the remaining 66 patients (92\%) underwent bilateral salpingo-oophorectomy plus hysterectomy, omentectomy, and/or peritoneal biopsies. Upon second review, 3 patients were found to have synchronous endometrial endometrioid carcinoma without myometrial invasion. In 1 , the ovarian carcinoma showed normal p53 and absence of MLH1/PMS2 expression, whereas the synchronous endometrial tumor had normal p53 and retained MMR. The other two cases had normal p53 and MMR expression in both tumors; however, the ovarian carcinoma was FIGO grade 2 and the endometrial carcinoma was FIGO grade 1.

Five patients $(7 \%)$ received neoadjuvant chemotherapy. Twenty-nine patients $(40 \%)$ received adjuvant platinum-based chemotherapy, either after initial surgery or after first recurrence; three patients $(4 \%)$ received adjuvant pelvic radiation. On the basis of clinical notes, none of the patients had family or personal history suggestive of $B R C A$ mutation carrier status. None of the patients in our cohort had undergone counseling or genetic testing for BRCA or Lynch syndrome at the time of last follow-up.

Distribution by FIGO histologic grade was as follows: $33(46 \%)$ grade $1 ; 24(33 \%)$ grade 2 ; and $15(21 \%)$ grade 3 . All cases had at least one confirmatory endometrioid feature; ovarian endometriosis was identified in 65/72 (90\%) cases. Endometrioid glandular morphology was consistently seen in all cases. Non-morular solid growth was present in varying proportions. Predominant transitional-like, papillary or micropapillary growth patterns were not observed in any of the cases included. The morphologic spectrum of ovarian endometrioid carcinoma in our series is depicted in Figures 1 and 2.

Figure 3 shows the case distribution following the molecular classifier. Among the 17 cases in p53abnormal group, 9 (53\%) tumors showed a strong and diffuse p53 staining pattern, whereas 8 tumors $(47 \%)$ showed complete absence of staining. Most tumors in this category were low grade $(12 / 17,71 \%)$. None of the cases showed concurrent p53/MMR abnormality or p53/POLE abnormality.

Among the six carcinomas in the MMR-abnormal group, four were MLH1-/PMS2-deficient, one was 


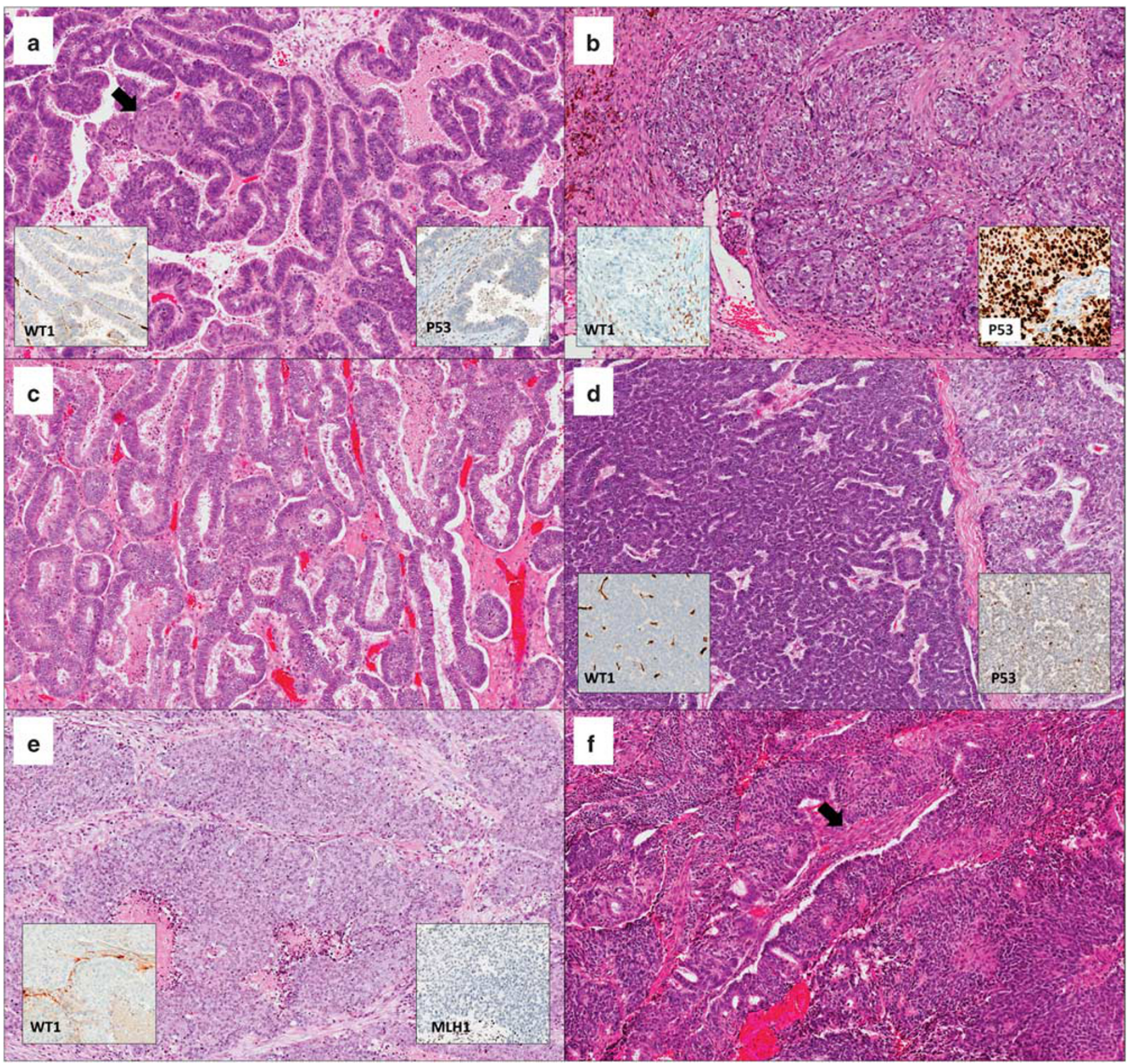

Figure 1 (a, b) Low-grade (a) and high-grade (b) ovarian endometrioid carcinomas, with negative WT1 and abnormal p53 expression (absent in a, overexpressed in b); both patients developed tumor recurrence. (c, d) Low-grade (c) and high-grade (d) ovarian endometrioid carcinomas with negative WT1 and wild-type p53 expression. (e) High-grade ovarian endometrioid carcinoma with MLH1 loss. (f) High-grade ovarian endometrioid carcinoma harboring a pathogenic POLE mutation. Arrows in (a and $\mathbf{f}$ ) indicate foci of squamous differentiation.

MSH2-/MSH6-deficient, and one was MSH6deficient only. A prominent intratumoral lymphocytic infiltrate was noted in two MMR-deficient tumors (one grade 2 and one grade 3 ).

The pathogenic or likely pathogenic variant mutations identified in the POLE gene are listed in Table 1. Of these, six constitute novel variants not previously described in ovarian endometrioid carcinoma. One tumor in the POLE-mutated group also showed loss of MLH1 and PMS2. A prominent intratumoral inflammatory component was observed in only one POLE-mutated case (grade 3).
Table 2 and Figure 4 show the distribution of clinical and pathologic variables by molecular group and grade. Compared to p53 wild-type and POLEmutated tumors, the p53-abnormal and MMRabnormal groups showed higher rates of ovarian surface involvement and advanced stage at presentation (FIGO stage II or more). The molecular subgroups did not show significant associations with FIGO grade, laterality, tumor size, or lymphovascular space invasion.

In all, 66 tumors were successfully tested for PTEN and ARID1A immunohistochemistry: 29 (44\%) 


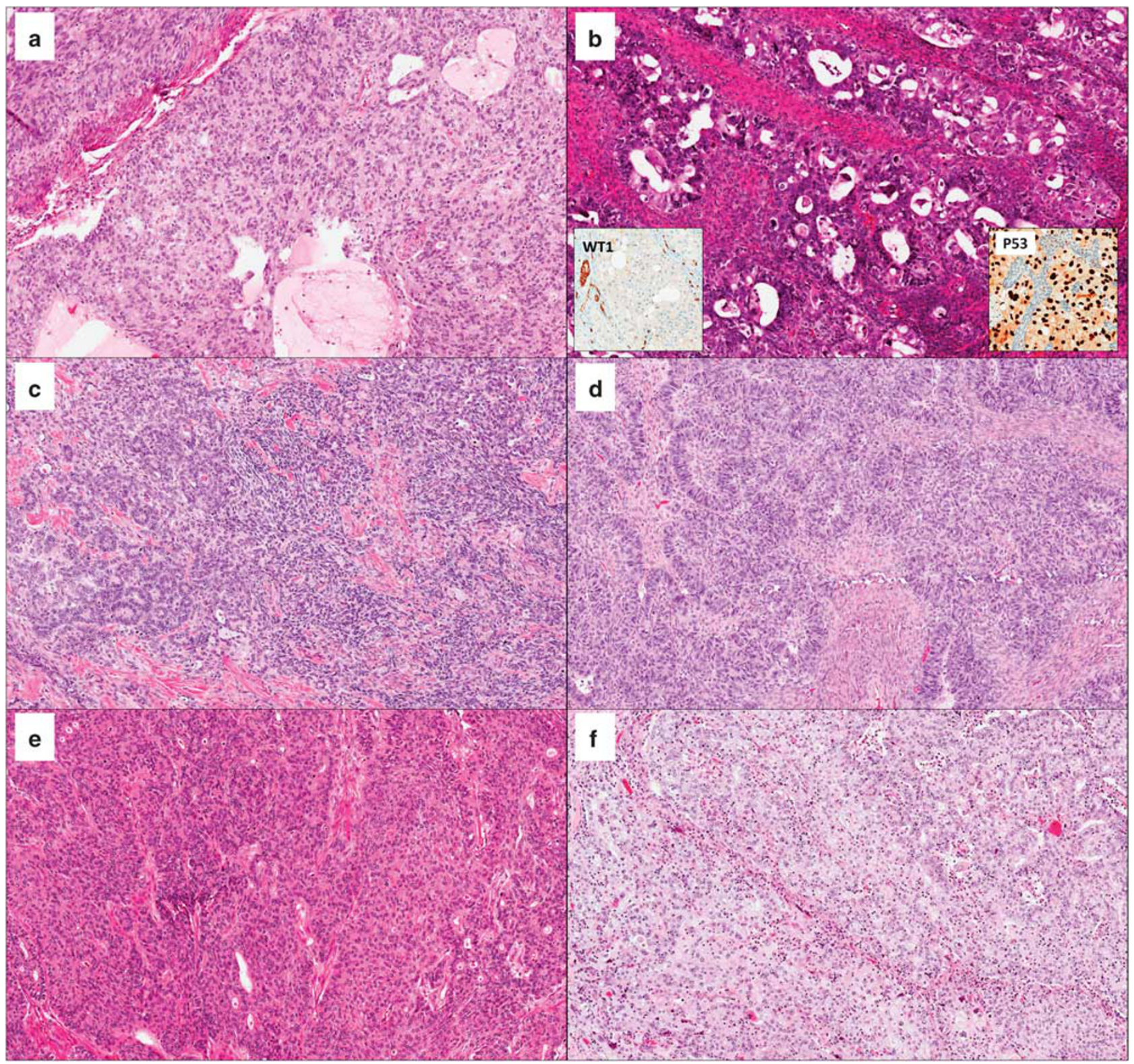

Figure 2 High-grade (FIGO 3) ovarian endometrioid carcinomas. (a-c) Tumors with abnormal p53 expression; these tumors are characterized by a predominant solid growth, admixed with endometrioid-type glands and exceeding $50 \%$ of the tumor volume; severe nuclear pleomorphism is noted in (a and b); inserts in (b) depict negative WT1 and p53 overexpression. (d, e) Tumors with wild-type p53; predominant non-morular solid growth; tumor in (d) had areas with sex cord appearance. (f) MMR-deficient tumor with glandular and solid architecture.

showed PTEN loss and $11(17 \%)$ showed ARID1A loss. Distribution among cases divided by molecular group and FIGO grade is depicted in Table 3. Most (86\%) POLE-mutated tumors showed PTEN loss, compared to $60 \%, 36 \%$, and $40 \%$ in the MMRabnormal, p53 wild-type, and p53-abnormal groups, respectively $(P=0.004)$. Similarly, $60 \%$ of MMRdeficient cases showed ARID1A loss, compared to 17,13 and $7 \%$ of POLE-mutated, p53 wild-type and p53-abnormal tumors, respectively $(P=0.02)$.

The median follow-up period was 62 months (mean 70 months, range 1-179). Univariate survival analysis results using log rank test are depicted in Table 4 and Figure 5. The four molecular categories obtained with the classifier correlated significantly with disease-free survival. P53-abnormal tumors carried a poor prognosis with a 5-year disease-free survival of $42 \%$. P53 wild-type cases had intermediate prognosis with a 5-year disease-free survival of $82 \%$. POLE-mutated and MMR-abnormal groups carried excellent prognosis with no recurrences or deaths reported (5-year disease-free survival $=$ $100 \%)$. The 5-year overall survival rate for p53abnormal, p53 wild-type, MMR-abnormal, and 


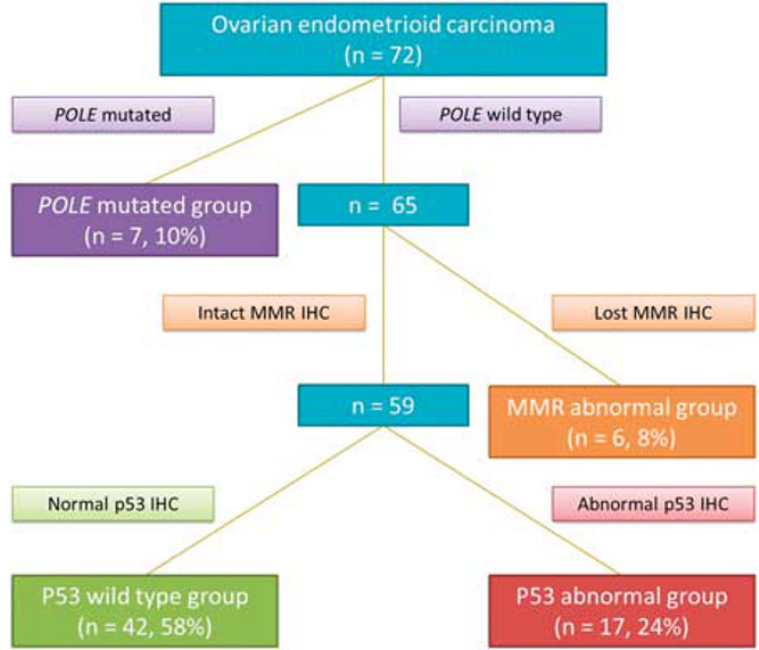

Figure 3 Diagram outlining patient distribution according to the molecular algorithm.

POLE-mutated groups was $76 \%, 86 \%, 100 \%$, and $100 \%$ respectively. Bilaterality, tumor grade, advanced stage, and lymphovascular space invasion all were associated with worse disease-free, overall, and disease-specific survival. Ovarian surface involvement correlated with disease-free and diseasespecific survival.

Table 5 shows multivariate analysis results. The molecular classification and stage were the only significant independent predictors of disease-free survival $(P=0.003$ and 0.026 , respectively, hazard ratio $=0.194$ and 5.432, respectively). In addition, the molecular classification was a near-significant predictor for overall survival $(P=0.051$, hazard ratio $=$ 0.375, 95\% confidence interval 0.140-1.002).

\section{Discussion}

Endometrioid carcinomas account for $\sim 10-25 \%$ of all ovarian carcinomas. ${ }^{7}$ It is predominantly seen in perimenopausal women in the context of endometriosis, which appears to act as a precursor lesion. ${ }^{8}$ Endometrioid carcinomas frequently harbor AT-rich interactive domain 1A (ARID1A) mutations leading to loss of ARID1A protein expression, ${ }^{9}$ as well as B-catenin (CTNNB1) somatic mutations, PTEN mutations, and microsatellite instability. ${ }^{10,11}$

Our aim was to apply the endometrial carcinoma molecular classification to ovarian endometrioid carcinoma using a validated classifier algorithm. The molecular classification for carcinoma of the endometrium represents a promising approach to stratify endometrial cancer patients into prognostically significant groups and therefore improve management. This positive impact in personalized care is particularly important in patients with high-grade endometrial carcinomas, a group with significant genomic heterogeneity and overlapping features with serous carcinoma ${ }^{12,13}$ and significant

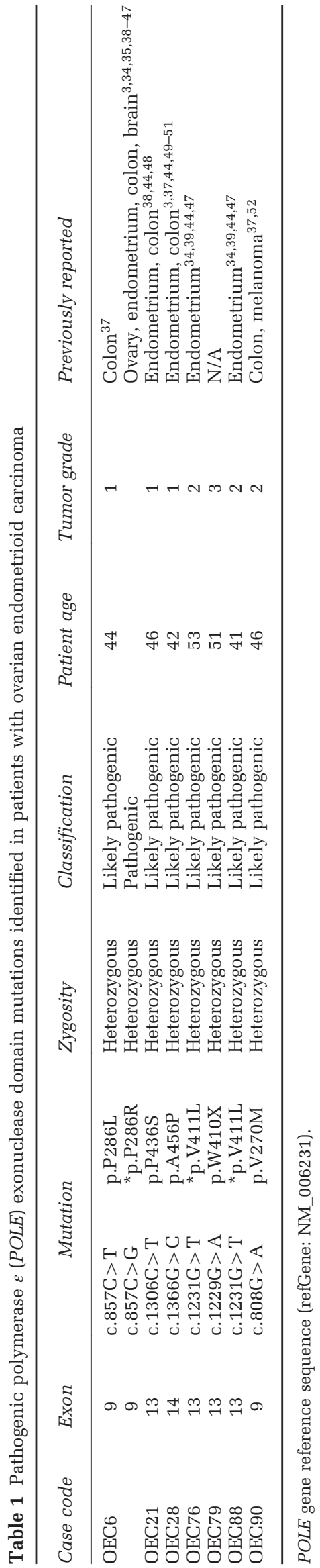


Table 2 Clinical and pathologic variables of patients with ovarian endometrioid carcinoma distributed by molecular group

\begin{tabular}{|c|c|c|c|c|c|}
\hline & & Group & & & \\
\hline & p53 abn & p53 wt & $M M R$ & POLE & \\
\hline $\begin{array}{l}\text { Number of } \\
\text { cases }\end{array}$ & $17(24 \%)$ & $42(58 \%)$ & $6(8 \%)$ & $7(10 \%)$ & \\
\hline Age (years) & & & & & \\
\hline $\begin{array}{l}\text { Median } \\
\text { (mean) }\end{array}$ & $55(60)$ & $55(55)$ & $55(53)$ & $46(46)$ & 0.147 \\
\hline Range & $27-91$ & $35-80$ & $33-65$ & $41-53$ & \\
\hline Tumor size ( & m) & & & & \\
\hline $\begin{array}{l}\text { Median } \\
\text { (mean) }\end{array}$ & $10(12.1)$ & $14(12.9)$ & $12(15.2)$ & $16(15.1)$ & 0.642 \\
\hline Range & $3.8-31$ & $1.9-27$ & $7.5-30$ & $8-20.5$ & \\
\hline Laterality & & & & & \\
\hline Unilateral & $12(71 \%)$ & $38(90 \%)$ & $5(83 \%)$ & $6(86 \%)$ & 0.294 \\
\hline Bilateral & $5(29 \%)$ & $4(10 \%)$ & $1(17 \%)$ & $1(14 \%)$ & \\
\hline FIGO grade & & & & & \\
\hline 1 & $4(24 \%)$ & $24(57 \%)$ & $2(33 \%)$ & $3(43 \%)$ & 0.362 \\
\hline 2 & 8 (47\%) & $11(26 \%)$ & 2 (33\%) & 3 (43\%) & \\
\hline 3 & $5(29 \%)$ & $7(17 \%)$ & $2(33 \%)$ & $1(14 \%)$ & \\
\hline$L V I$ & & & & & \\
\hline Absent & $13(77 \%)$ & $38(90 \%)$ & $3(50 \%)$ & $6(86 \%)$ & 0.074 \\
\hline Present & $4(23 \%)$ & $4(10 \%)$ & $3(50 \%)$ & $1(14 \%)$ & \\
\hline$E M S$ & & & & & \\
\hline Absent & $2(12 \%)$ & $5(12 \%)$ & 0 & $1(14 \%)$ & 0.836 \\
\hline Present & $15(88 \%)$ & $37(88 \%)$ & $6(100 \%)$ & $6(86 \%)$ & \\
\hline Surface invo & vement & & & & \\
\hline Absent & 8 (47\%) & $31(74 \%)$ & $1(17 \%)$ & $5(72 \%)$ & 0.029 \\
\hline Present & 8 (47\%) & $11(26 \%)$ & $5(83 \%)$ & $2(28 \%)$ & \\
\hline Unknown & $1(6 \%)$ & 0 & 0 & 0 & \\
\hline FIGO stage & & & & & \\
\hline I & 8 (47\%) & $31(74 \%)$ & $1(17 \%)$ & $6(86 \%)$ & 0.015 \\
\hline II-IV & 8 (47\%) & $11(26 \%)$ & $5(83 \%)$ & $1(14 \%)$ & \\
\hline Unknown & $1(6 \%)$ & 0 & 0 & 0 & \\
\hline Follow-up pe & riod (mont) & $h s)$ & & & \\
\hline $\begin{array}{l}\text { Median } \\
\text { (mean) }\end{array}$ & $35(48)$ & $65(78)$ & $84(75)$ & $63(73)$ & 0.133 \\
\hline Range & $1-128$ & $3-179$ & $37-98$ & $32-168$ & \\
\hline Disease statu & $s$ at last fol & llow-up & & & \\
\hline ANED & $6(35 \%)$ & $35(83 \%)$ & $6(100 \%)$ & $7(100 \%)$ & Tables 3 \\
\hline AWD & $6(35 \%)$ & $1(2.5 \%)$ & 0 & 0 & and 4 \\
\hline DOD & $4(24 \%)$ & $5(12 \%)$ & 0 & 0 & \\
\hline DOC & $1(6 \%)$ & $1(2.5 \%)$ & 0 & 0 & \\
\hline
\end{tabular}

Abbreviations: Abn, abnormal; ANED, alive with no evidence of disease; AWD, alive with disease; DOC, dead of other causes; DOD, dead of disease; EMS, endometriosis; FIGO, International Federation of Gynecology and Obstetrics; LVI, lymphovascular space invasion; MMR, mismatch repair; wt, wild type.

${ }^{\mathrm{a}} P$-values were obtained using $\chi^{2}$-test for categorical variables and oneway ANOVA for continuous variables.

interobserver variation in its diagnosis. ${ }^{14,15}$ In contrast to endometrial carcinoma, ovarian carcinoma subtypes have more distinct biologic and clinical differences, including stage, survival, and management. ${ }^{16,17}$ Moreover, ovarian carcinoma subtyping has been demonstrated to have high reproducibility, ${ }^{7,18}$ which greatly improves with the use of a targeted immunohistochemistry panel. ${ }^{19-21}$ Although the existence of a high-grade ovarian endometrioid type has been debated, the evidence shows that a small, but significant subset of ovarian endometrioid carcinomas are indeed high grade. ${ }^{22,23}$ Moreover, low-grade endometrial tumors have potential for adverse outcome, as seen in our study. For these reasons, ancillary testing has a role in the work-up of ovarian endometrioid carcinomas beyond tumor type and grade, akin to endometrial carcinoma.

While histopathology and protein expression optimally separate ovarian carcinoma into clinically different types, further proteomic and genomic characterization may provide useful prognostic information. This is important for the ovarian endometrioid carcinoma subtype, which, similar to its uterine counterpart, represents a heterogeneous group of tumors. Although most ovarian endometrioid carcinomas are low risk and early stage, a subset has high-risk features and behaves aggressively, as seen in our study. We demonstrate that p53, MMR, and POLE analysis can be used to assess prognosis in ovarian endometrioid carcinoma as surrogates of the molecular profile, similar to endometrial endometrioid carcinoma.

Misclassification of other types of ovarian carcinoma, especially serous, as endometrioid is documented; in fact, it has been postulated that the majority of high-grade endometrioid carcinomas represent serous carcinomas with variant (solid, endometrioid, and transitional-SET) morphology. ${ }^{23}$ To rule out mimics of endometrioid morphology, expert pathologist review was carried to confirm an endometrioid phenotype and exclude serous carcinomas with SET morphology. Histopathologic analysis showed a spectrum akin to that observed in endometrial tumors, characterized by endometrioid-type glands with smooth luminal borders and columnar cells. High-grade cases had non-morular solid growth exceeding $50 \%$ of the tumor volume; nuclear pleomorphism ranged from mild to severe. Moreover, all carcinomas in our study had at least one confirmatory endometrioid feature as an inclusion criterion (endometriosis, endometrioid adenofibroma background, and/or squamous, mucinous, or secretory metaplasia). In addition, we only included tumors that were negative for WT1 and Napsin-A by immunohistochemistry. WT1-positive ovarian endometrioid carcinomas have a gene expression profile similar to serous carcinomas, and likely represent serous tumors misclassified as or mimicking endometrioid morphology. ${ }^{22,23}$ Thus, under current immunohistochemical algorithms ovarian endometrioid carcinoma is defined as hormone receptor-positive, Napsin-Anegative and WT1-negative. ${ }^{19,20,24}$ Some authors have reported WT1 expression in up to $30 \%$ of endometrioid carcinomas, ${ }^{25,26}$ although misclassification of 

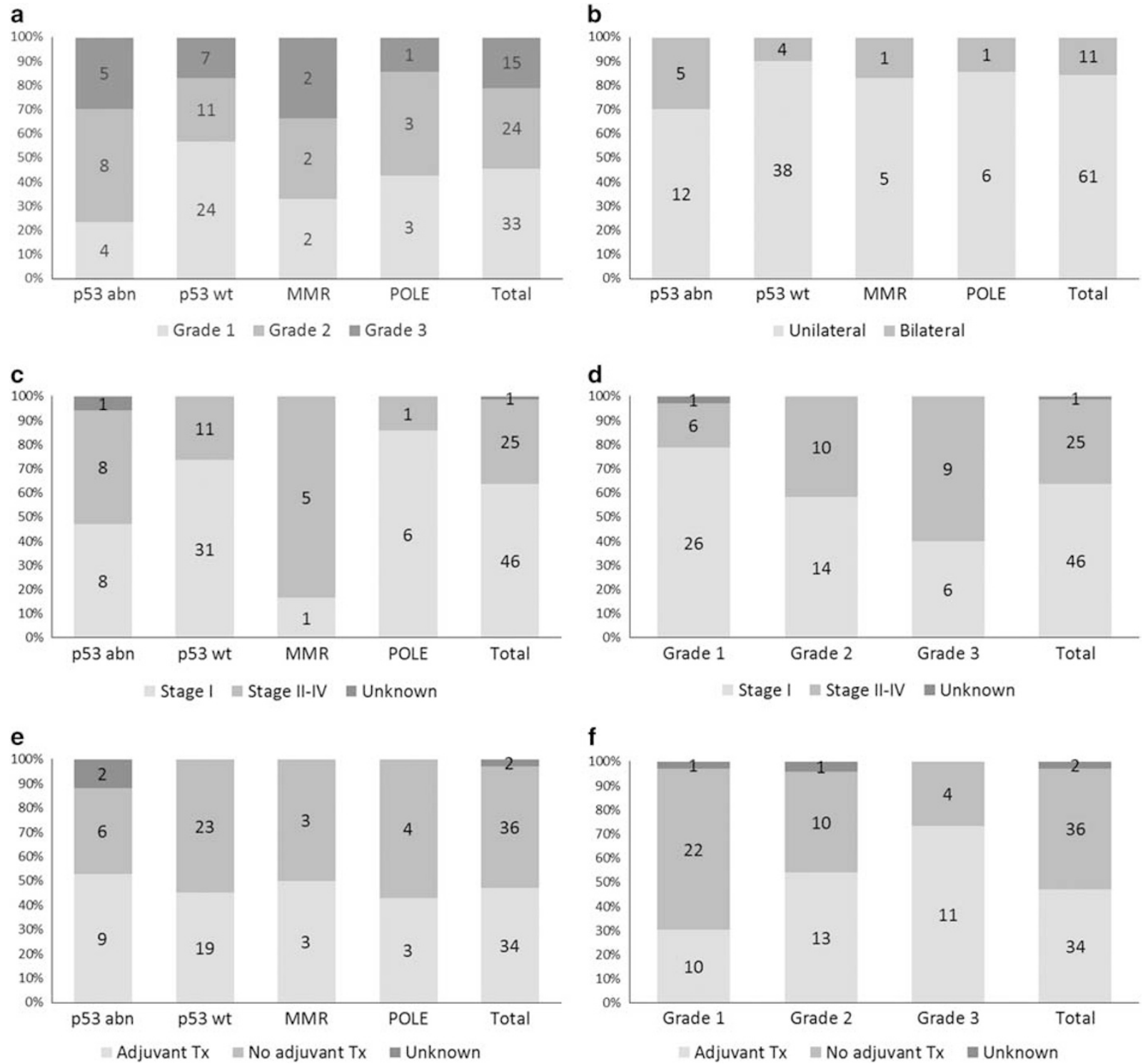

Figure 4 Case distribution according to molecular group versus histologic grade (a), molecular group versus tumor laterality (b), molecular group versus stage (c), grade versus stage (d), molecular group versus adjuvant treatment (e), and grade versus adjuvant treatment (f). Numbers in each bar represent absolute case numbers.

Table 3 ARID1A and PTEN loss by immunohistochemistry in ovarian endometrioid carcinomas classified by molecular group and grade

\begin{tabular}{lrr}
\hline & ARID1A & \multicolumn{1}{c}{ PTEN } \\
\hline p53 abn $(n=15)$ & $1(7 \%)$ & $6(40 \%)$ \\
p53 wt $(n=39)$ & $5(13 \%)$ & $14(36 \%)$ \\
MMR $(n=5)$ & $3(60 \%)$ & $3(60 \%)$ \\
POLE $(n=7)$ & $2(29 \%)$ & $6(86 \%)$ \\
Grade $1(n=29)$ & $5(17 \%)$ & $12(41 \%)$ \\
Grade 2 $(n=23)$ & $4(17 \%)$ & $10(43 \%)$ \\
Grade 3 $(n=14)$ & $2(14 \%)$ & $7(50 \%)$ \\
Total $(n=66)$ & $11(17 \%)$ & $29(44 \%)$ \\
\hline
\end{tabular}

high-grade serous carcinomas as endometrioid in such studies is probable. ${ }^{22}$ On the basis of the criteria outlined above, tumor type misclassification in our cohort is considered unlikely.
The classifier used in this study allowed us to divide ovarian endometrioid carcinomas into four groups with distinct overall and disease-free survival. Among them, the 'p53-abnormal' group carried the worst prognosis. Abnormal p53 expression has been previously reported in $13-39 \%$ of ovarian endometrioid carcinomas, usually associated with grade 3 and advanced-stage tumors. ${ }^{22,27,28}$ In one cohort of 29 ovarian endometrioid carcinomas, p53 overexpression was present in 6 tumors (21\%), most of them high grade. ${ }^{29}$ We found a similar proportion of carcinomas with abnormal p53 expression (24\%). Remarkably, in our study 4 out of the 17 tumors in the p53-abnormal group were grade 1; of these, 2 patients developed tumor recurrence. Moreover, 8 subjects $(47 \%)$ had grade 2 tumors, of which two recurred and 2 died of disease. These observations 
Table 4 Univariate analysis of survival in patients with ovarian endometrioid carcinoma

\begin{tabular}{|c|c|c|c|c|c|c|c|c|c|c|}
\hline & \multicolumn{4}{|c|}{$D F S$} & \multicolumn{3}{|c|}{ OS } & \multicolumn{3}{|c|}{$D S S$} \\
\hline & Total & Event & $\%$ of event & $P$ & Event & $\%$ of event & $P$ & Event & $\%$ of event & $P$ \\
\hline \multicolumn{11}{|c|}{ Molecular group } \\
\hline p53 abn & 17 & 10 & 58.8 & $<0.001$ & 5 & 29.4 & 0.094 & 4 & 23.5 & 0.214 \\
\hline p53 wt & 42 & 6 & 14.3 & & 6 & 14.3 & & 5 & 11.9 & \\
\hline MMR & 6 & 0 & 0.0 & & 0 & 0.0 & & 0 & 0.0 & \\
\hline POLE & 7 & 0 & 0.0 & & 0 & 0.0 & & 0 & 0.0 & \\
\hline \multicolumn{11}{|l|}{ Laterality } \\
\hline Unilateral & 61 & 11 & 18.0 & 0.010 & 7 & 11.5 & 0.003 & 6 & 9.8 & 0.028 \\
\hline Bilateral & 11 & 5 & 45.5 & & 4 & 36.4 & & 3 & 27.3 & \\
\hline \multicolumn{11}{|c|}{ Surface involvement } \\
\hline Absent & 45 & 5 & 11.1 & 0.001 & 5 & 11.1 & 0.077 & 3 & 6.7 & 0.024 \\
\hline Present & 26 & 11 & 42.3 & & 6 & 23.1 & & 6 & 23.1 & \\
\hline \multicolumn{11}{|l|}{ FIGO grade } \\
\hline 1 & 33 & 3 & 9.1 & 0.006 & 2 & 6.1 & 0.009 & 1 & 3.0 & 0.003 \\
\hline 2 & 24 & 6 & 25.0 & & 4 & 16.7 & & 3 & 12.5 & \\
\hline 3 & 15 & 7 & 46.7 & & 5 & 33.3 & & 5 & 33.3 & \\
\hline \multicolumn{11}{|l|}{$L V I$} \\
\hline 0 & 60 & 10 & 16.7 & 0.004 & 7 & 11.7 & 0.019 & 5 & 8.3 & 0.007 \\
\hline 1 & 12 & 6 & 50.0 & & 4 & 33.3 & & 4 & 33.3 & \\
\hline \multicolumn{11}{|l|}{ FIGO stage } \\
\hline I & 46 & 4 & 8.7 & $<0.001$ & 4 & 8.7 & 0.011 & 2 & 4.3 & 0.002 \\
\hline II-IV & 25 & 12 & 48.0 & & 7 & 28.0 & & 7 & 28.0 & \\
\hline
\end{tabular}

Abbreviations: DFS, disease-free survival; DSS, disease-specific survival; LVI, lymphovascular space invasion; OS, overall survival.

a

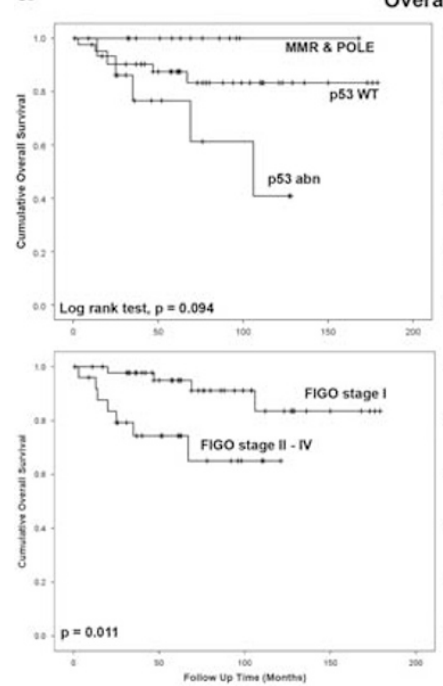

Overall Survival

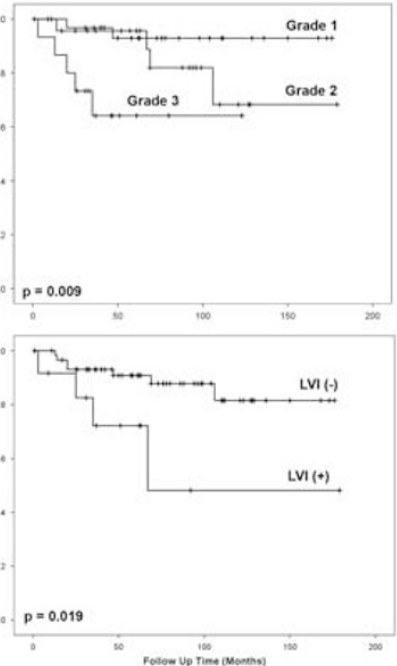

b

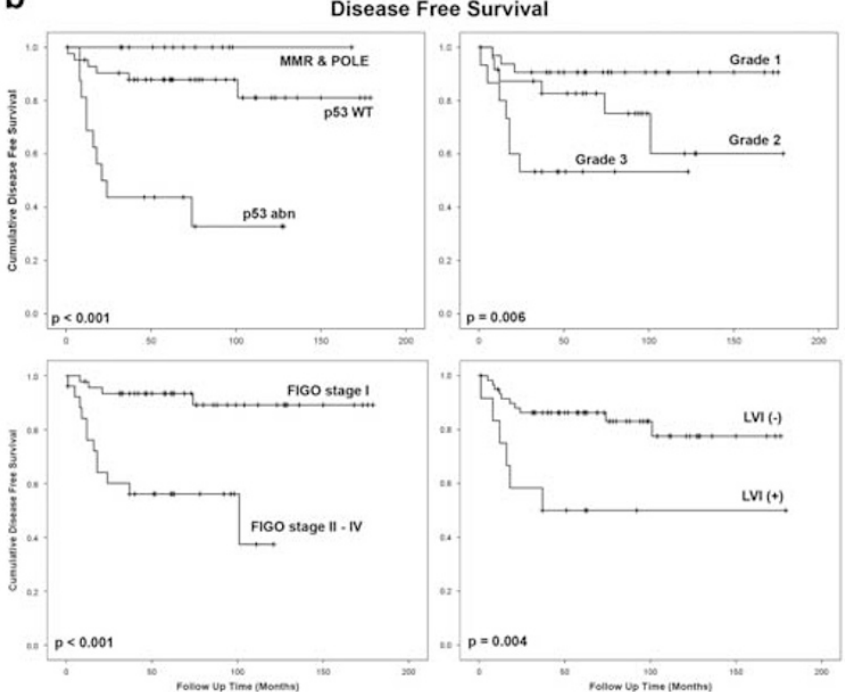

Figure 5 Kaplan-Meier survival analysis curves for disease-free survival (a) and overall survival (b). Each composite displays survival according to molecular subgroup (upper left), tumor grade (upper right), stage (lower left), and lymphovascular space invasion (LVI, lower right).

highlight the potential role of p53 as a marker of adverse behavior, independent of traditional parameters such as tumor grade.

Defective mismatch repair protein immunohistochemistry has been reported in 10-14\% of ovarian endometrioid carcinomas, ${ }^{30-32}$ similar to the prevalence seen in our cohort (6/72 cases, $8 \%)$. Over
$50 \%$ of MMR-deficient ovarian endometrioid carcinomas have loss of MSH2 and/or MSH6. ${ }^{31,33}$ In addition, a significant subset of these tumors has been related to Lynch syndrome. ${ }^{32}$ Previous studies have failed to demonstrate a significant correlation between MMR status and survival, possibly due to the low prevalence of MMR deficiency in 
Table 5 Multivariate analysis of survival using Cox proportional hazards models in patients with ovarian endometrioid carcinoma

\begin{tabular}{llllr}
\hline & & & \multicolumn{2}{c}{$95.0 \%$ CI for HR } \\
\cline { 4 - 5 } & P-value & HR & & \\
\cline { 4 - 5 } & & & Lower & Upper \\
\hline DFS & & & & \\
Molecular group & 0.003 & 0.194 & 0.066 & 0.572 \\
Laterality & 0.367 & 1.850 & 0.486 & 7.038 \\
Surface & 0.773 & 1.265 & 0.257 & 6.215 \\
Grade & 0.572 & 1.271 & 0.554 & 2.917 \\
LVI & 0.739 & 1.256 & 0.327 & 4.824 \\
Stage & 0.026 & 5.432 & 1.227 & 24.051 \\
& & & & \\
OS & & & & \\
Molecular group & 0.051 & 0.375 & 0.140 & 1.002 \\
Laterality & 0.081 & 3.960 & 0.842 & 18.628 \\
Grade & 0.215 & 1.857 & 0.698 & 4.940 \\
LVI & 0.976 & 1.026 & 0.188 & 5.590 \\
Stage & 0.084 & 3.718 & 0.839 & 16.482 \\
DSS & & & & \\
Molecular group & 0.154 & 0.485 & 0.180 & 1.310 \\
Laterality & 0.251 & 2.796 & 0.482 & 16.207 \\
Surface & 0.487 & 0.464 & 0.053 & 4.053 \\
Grade & 0.110 & 2.665 & 0.800 & 8.878 \\
LVI & 0.833 & 1.237 & 0.171 & 8.961 \\
Stage & 0.043 & 9.630 & 1.079 & 85.953 \\
& & & & \\
\hline
\end{tabular}

Abbreviations: CI, confidence interval; DFS, disease-free survival; DSS, disease-specific survival; HR, hazard ratio; LVI, lymphovascular space invasion; OS, overall survival.

endometrioid ovarian carcinoma. Nonetheless, one study reported death in only $2 / 14(14 \%)$ of patients with MMR-deficient tumors. ${ }^{30}$ Our results show a good prognosis in the MMR-deficient group with no recurrences or death, suggesting that MMR status may also have value in patient prognostication. Interestingly, morphologic characteristics of MMRdeficient endometrial carcinomas such as a peri- and intratumoral dense lymphoplasmacytic infiltrate and syncytial-like appearance were not prevalent in our cohort, being observed in only 2 of 6 MMR-deficient ovarian tumors (1 FIGO grade 2 and 1 FIGO grade 3 ).

Mutations in the exonuclease domain of POLE have been found in $7-8 \%$ of endometrial carcinomas. $^{1,3}$ Tumors with this alteration are described as 'ultramutated', as POLE mutations lead to an increased $\mathrm{C}$ to $\mathrm{A}$ transversion frequency in multiple genes. This group of carcinomas is characterized by an improved progression-free survival compared to other genomic groups, regardless of tumor grade. ${ }^{1,34}$ POLE exonuclease domain mutations were reported in 4 out of $89(4.5 \%)$ ovarian endometrioid carcinomas in one study, all missense point mutations affecting previously known hotspots (P286R and V411L). ${ }^{35}$ Of note, three of these tumors were FIGO grade 1 and one was FIGO grade 2; all four followed an indolent clinical course. The POLEmutated group comprised $10 \%$ of our study cohort (7 out of 72 patients). Remarkably, all patients in the POLE-mutated group were alive with no evidence of disease at time of follow-up, indicating that POLE has an important prognostic role in ovarian endometrioid carcinomas similar to endometrial cancer. Importantly, one tumor in this group was grade 3 and three were grade 2, suggesting that POLE mutations are not exclusive of low-grade (FIGO grade 1) tumors in the ovary. Indeed, POLE mutations (somatic and germline) have been reported in up to $29 \%$ FIGO grade 3 endometrial endometrioid carcinomas. ${ }^{34}$ Similarly, in a separate study $63 \%$ of POLE-mutated endometrial carcinomas were FIGO grade $3 .^{36}$ This study also noted a prominent lymphophasmacytic infiltrate in POLE-mutated endometrial carcinomas; we observed such finding in only one FIGO grade 3 POLE-mutated ovarian endometrioid carcinoma. Loss of PTEN expression was seen in most POLE-mutated cases, at a higher rate compared to the other groups. This is consistent with previous literature showing frequent concurrent POLE and PTEN mutations in endometrial cancer. ${ }^{1,3}$

Six POLE mutation variants were classified as either likely pathogenic or pathogenic. All pathogenic/likely pathogenic variants except one have been previously reported in the literature, mostly in endometrial and colorectal carcinomas (Table 1). Of note, only one of the variants found in our cohort has been previously reported in ovarian endometrioid carcinoma (c.857C $>$ G, p.P286R). ${ }^{35}$ The c.1229G $>$ A variant, previously not reported, produces a nonsense mutation near the $5^{\prime}$-end of the exonuclease domain leading to a premature stop codon at position 410 (p.W410X), which predictably causes protein truncation and loss of function. Typically, missense mutations have been reported in the exonuclease domain as the type of variant expected to cause tumorigenesis. Thus, this variant is best classified as likely pathogenic. Loss of heterozygosity or allelic heterogeneity may be responsible for the clinical manifestations in this case.

In addition to the pathogenic/likely pathogenic variants described above, 20 variants of uncertain significance were identified in the POLE gene in this cohort (Supplementary Table 1). Although several in silico prediction software (AlighGVGD, SIFT, PolyPhen, Mutation TASTER, etc) suggest these variants may be deleterious, there was limited evidence either from the literature or other database sources to determine whether or not these variants are clinically significant according to the ACMG 2015 guidelines. Thus, the proportion of mutations in the POLE gene in patients with endometrioid carcinomas may be higher than what we report here. Further investigation into the mutational spectrum of the $P O L E$ gene is required to evaluate the clinical significance of these variants.

In summary, we demonstrate that ovarian endometrioid carcinoma is a heterogeneous group of tumors, harboring alterations in p53, MMR, and POLE. Such abnormalities are associated with patient outcome, and their detection can be useful 
to subclassify ovarian endometrioid tumors into prognostically significant groups analogous to endometrial cancer. The value and feasibility of the proposed molecular algorithm seem promising, as the proposed markers are either already available in clinical laboratories or on the verge to enter into routine practice.

\section{Acknowledgments}

We thank Nim Li from Sunnybrook Health Sciences Centre for the construction and maintenance of the Biomatrix database; Kai Xuan Leong, Kathleen-Rose Zakoor, Sharon Crafter, Nahida El Warry, and Anna Kiselova from the Advanced Molecular Diagnostic Laboratory at Mount Sinai Hospital for DNA extraction, PCR, sequencing, analysis, and interpretation of results; and Tara Paton and Guillermo Casallo of The Centre for Applied Genomics, The Hospital for Sick Children (Toronto, Canada) for their assistance with PCR and sequencing.

\section{Disclosure/conflict of interest}

The authors declare no conflict of interest.

\section{References}

1 Kandoth C, Schultz N, Cherniack AD, et al. Integrated genomic characterization of endometrial carcinoma. Nature 2013;497:67-73.

2 McConechy MK, Ding J, Cheang MCU, et al. Use of mutation profiles to refine the classification of endometrial carcinomas. J Pathol 2012;228:20-30.

3 Talhouk A, McConechy MK, Leung S, et al. A clinically applicable molecular-based classification for endometrial cancers. Br J Cancer 2015;113:299-310.

4 Talhouk A, McConechy MK, Leung S, et al. Confirmation of ProMisE: a simple, genomics-based clinical classifier for endometrial cancer. Cancer 2017;123: 802-813.

5 Richards S, Aziz N, Bale S, et al. Standards and guidelines for the interpretation of sequence variants: a joint consensus recommendation of the American College of Medical Genetics and Genomics and the Association for Molecular Pathology. Genet Med 2015;17:405-424.

6 Lerner-Ellis J, Wang M, White S, et al. Canadian Open Genetics Repository (COGR): a unified clinical genomics database as a community resource for standardising and sharing genetic interpretations. J Med Genet 2015;52:438-445.

7 Gilks CB, Ionescu DN, Kalloger SE, et al. Tumor cell type can be reproducibly diagnosed and is of independent prognostic significance in patients with maximally debulked ovarian carcinoma. Hum Pathol 2008;39: 1239-1251.

8 Jiang X, Morland SJ, Hitchcock A, et al. Allelotyping of endometriosis with adjacent ovarian carcinoma reveals evidence of a common lineage. Cancer Res 1998;58: 1707-1712.
9 Wiegand KC, Shah SP, Al-Agha OM, et al. ARID1A mutations in endometriosis-associated ovarian carcinomas. N Engl J Med 2010;363:1532-1543.

10 Catasús L, Bussaglia E, Rodrguez I, et al. Molecular genetic alterations in endometrioid carcinomas of the ovary: similar frequency of beta-catenin abnormalities but lower rate of microsatellite instability and PTEN alterations than in uterine endometrioid carcinomas. Hum Pathol 2004;35:1360-1368.

11 McConechy MK, Ding J, Senz J, et al. Ovarian and endometrial endometrioid carcinomas have distinct CTNNB1 and PTEN mutation profiles. Mod Pathol 2014;27:128-134.

12 Hussein YR, Broaddus R, Weigelt B, et al. The genomic heterogeneity of FIGO grade 3 endometrioid carcinoma impacts diagnostic accuracy and reproducibility. Int J Gynecol Pathol 2016;35:16-24.

13 Hoang LN, McConechy MK, Köbel M, et al. Histotypegenotype correlation in 36 high-grade endometrial carcinomas. Am J Surg Pathol 2013;37:1421-1432.

14 Han G, Sidhu D, Duggan MA, et al. Reproducibility of histological cell type in high-grade endometrial carcinoma. Mod Pathol 2013;26:1594-1604.

15 Gilks CB, Oliva E, Soslow RA. Poor interobserver reproducibility in the diagnosis of high-grade endometrial carcinoma. Am J Surg Pathol 2013;37:874-881.

16 Köbel M, Kalloger SE, Huntsman DG, et al. Differences in tumor type in low-stage versus high-stage ovarian carcinomas. Int J Gynecol Pathol 2010;29:203-211.

17 Köbel M, Kalloger SE, Boyd N, et al. Ovarian carcinoma subtypes are different diseases: implications for biomarker studies. PLoS Med 2008;5:e232.

18 Köbel M, Kalloger SE, Baker PM, et al. Diagnosis of ovarian carcinoma cell type is highly reproducible: a transcanadian study. Am J Surg Pathol 2010;34:984-993.

19 Köbel M, Bak J, Bertelsen BI, et al. Ovarian carcinoma histotype determination is highly reproducible, and is improved through the use of immunohistochemistry. Histopathology 2014;64:1004-1013.

20 Kalloger SE, Köbel M, Leung S, et al. Calculator for ovarian carcinoma subtype prediction. Mod Pathol 2011;24:512-521.

21 Kommoss S, Gilks CB, Kommoss F, et al. Accelerating type-specific ovarian carcinoma research: Calculator for Ovarian Subtype Prediction (COSP) is a reliable high-throughput tool for case review. Histopathology 2013;63:704-712.

22 Madore J, Ren F, Filali-Mouhim A, et al. Characterization of the molecular differences between ovarian endometrioid carcinoma and ovarian serous carcinoma. J Pathol 2010;220:392-400.

23 Lim D, Murali R, Murray MP, et al. Morphological and immunohistochemical reevaluation of tumors initially diagnosed as ovarian endometrioid carcinoma with emphasis on high-grade tumors. Am J Surg Pathol 2016;40:302-312.

24 Acs G, Pasha T, Zhang PJ. WT1 is differentially expressed in serous, endometrioid, clear cell, and mucinous carcinomas of the peritoneum, fallopian tube, ovary, and endometrium. Int J Gynecol Pathol 2004;23:110-118.

25 Cathro HP, Stoler MH. The utility of calretinin, inhibin, and WT1 immunohistochemical staining in the differential diagnosis of ovarian tumors. Hum Pathol 2005;36:195-201.

26 Waldstrøm M, Grove A. Immunohistochemical expression of wilms tumor gene protein in different histologic 
subtypes of ovarian carcinomas. Arch Pathol Lab Med 2005;129:85-88.

27 Leng J, Lang J, Shen K, et al. Overexpression of p53, EGFR, c-erbB2 and c-erbB3 in endometrioid carcinoma of the ovary. Chin Med Sci J 1997;12:67-70.

28 Sawairiitoh $\mathrm{M}$, Imai A, Murase $\mathrm{T}$, et al. Different patterns of p53 gene-mutations in endometrial carcinomas and endometrioid carcinomas of the ovary. Int J Oncol 1994;5:583-588.

29 Geyer JT, López-García MA, Sánchez-Estevez C, et al. Pathogenetic pathways in ovarian endometrioid adenocarcinoma: a molecular study of 29 cases. Am J Surg Pathol 2009;33:1157-1163.

30 Aysal A, Karnezis A, Medhi I, et al. Ovarian endometrioid adenocarcinoma: incidence and clinical significance of the morphologic and immunohistochemical markers of mismatch repair protein defects and tumor microsatellite instability. Am J Surg Pathol 2012;36: 163-172.

31 Rambau PF, Duggan MA, Ghatage P, et al. Significant frequency of MSH2/MSH6 abnormality in ovarian endometrioid carcinoma supports histotype-specific Lynch syndrome screening in ovarian carcinomas. Histopathology 2016;69:288-297.

32 Vierkoetter KR, Ayabe AR, VanDrunen M, et al. Lynch Syndrome in patients with clear cell and endometrioid cancers of the ovary. Gynecol Oncol 2014;135:81-84.

33 Huang $\mathrm{H}-\mathrm{N}$, Lin M-C, Tseng L-H, et al. Ovarian and endometrial endometrioid adenocarcinomas have distinct profiles of microsatellite instability, PTEN expression, and ARID1A expression. Histopathology 2015;66:517-528.

34 Wong A, Kuick CH, Wong WL, et al. Mutation spectrum of POLE and POLD1 mutations in South East Asian women presenting with grade 3 endometrioid endometrial carcinomas. Gynecol Oncol 2016;141: 113-120.

35 Hoang LN, McConechy MK, Köbel M, et al. Polymerase epsilon exonuclease domain mutations in ovarian endometrioid carcinoma. Int J Gynecol Cancer 2015;25: 1187-1193.

36 Bakhsh S, Kinloch M, Hoang LN, et al. Histopathological features of endometrial carcinomas associated with POLE mutations: implications for decisions about adjuvant therapy. Histopathology 2016;68: 916-924.

37 Stenzinger A, Pfarr N, Endris V, et al. Mutations in POLE and survival of colorectal cancer patients-link to disease stage and treatment. Cancer Med 2014;3: 1527-1538.

38 Ahn S-M, Ansari AA, Kim J, et al. The somatic POLE P286R mutation defines a unique subclass of colorectal cancer featuring hypermutation, representing a potential genomic biomarker for immunotherapy. Oncotarget 2016;7:68638-68649.
39 van Gool IC, Eggink FA, Freeman-Mills L, et al. POLE proofreading mutations elicit an antitumor immune response in endometrial cancer. Clin Cancer Res 2015;21:3347-3355.

40 Erson-Omay EZ, Çağlayan AO, Schultz N, et al. Somatic POLE mutations cause an ultramutated giant cell high-grade glioma subtype with better prognosis. Neuro-Oncol 2015;17:1356-1364.

41 Church DN, Stelloo E, Nout RA, et al. Prognostic significance of POLE proofreading mutations in endometrial cancer. J Natl Cancer Inst 2015;107:402.

42 Jones S, Stransky N, McCord CL, et al. Genomic analyses of gynaecologic carcinosarcomas reveal frequent mutations in chromatin remodelling genes. Nat Commun 2014;5:5006.

43 Billingsley CC, Cohn DE, Mutch DG, et al. Polymerase $\varepsilon$ (POLE) mutations in endometrial cancer: clinical outcomes and implications for Lynch syndrome testing. Cancer 2015;121:386-394.

44 Meng B, Hoang LN, McIntyre JB, et al. POLE exonuclease domain mutation predicts long progression-free survival in grade 3 endometrioid carcinoma of the endometrium. Gynecol Oncol 2014;134:15-19.

45 Mouradov D, Sloggett C, Jorissen RN, et al. Colorectal cancer cell lines are representative models of the main molecular subtypes of primary cancer. Cancer Res 2014;74:3238-3247.

46 Abaan OD, Polley EC, Davis SR, et al. The exomes of the NCI-60 panel: a genomic resource for cancer biology and systems pharmacology. Cancer Res 2013;73:4372-4382.

47 Kane DP, Shcherbakova PV. A common cancerassociated DNA polymerase $\varepsilon$ mutation causes an exceptionally strong mutator phenotype, indicating fidelity defects distinct from loss of proofreading. Cancer Res 2014;74:1895-1901.

48 Spier I, Holzapfel S, Altmüller J, et al. Frequency and phenotypic spectrum of germline mutations in POLE and seven other polymerase genes in 266 patients with colorectal adenomas and carcinomas. Int J Cancer 2015;137:320-331.

49 Shinbrot E, Henninger EE, Weinhold N, et al. Exonuclease mutations in DNA polymerase epsilon reveal replication strand specific mutation patterns and human origins of replication. Genome Res 2014;24: 1740-1750.

50 Jansen AM, van Wezel T, van den Akker BE, et al. Combined mismatch repair and POLE/POLD1 defects explain unresolved suspected Lynch syndrome cancers. Eur J Hum Genet 2016;24:1089-1092.

51 Church DN, Briggs SEW, Palles C, et al. DNA polymerase $\varepsilon$ and $\delta$ exonuclease domain mutations in endometrial cancer. Hum Mol Genet 2013;22:2820-2828.

52 Aoude LG, Heitzer E, Johansson P, et al. POLE mutations in families predisposed to cutaneous melanoma. Fam Cancer 2015;14:621-628.

Supplementary Information accompanies the paper on Modern Pathology website (http://www.nature.com/ modpathol) 